\title{
The first self-endothelialized titanium-coated glutaraldehyde-fixed heart valve prosthesis within systemic circulation
}

\author{
Norbert W. Guldner, MD, ${ }^{\text {a }}$ Inka Jasmund, MS, ${ }^{a}$ Hanngörg Zimmermann, MS, ${ }^{\text {b }}$ Markus Heinlein, MS, ${ }^{\text {b }}$ \\ Britta Girndt, MS, ${ }^{\mathrm{b}}$ Martin Großherr, MD, ${ }^{\mathrm{c}}$ Mathias Klinger, MD, ${ }^{\mathrm{d}}$ and Hans H. Sievers, MD, ${ }^{\mathrm{a}}$ \\ Nürnberg and Lübeck, Germany
}

At present, 275000 heart valve replacements are performed annually world wide. Roughly $60 \%$ are glutaraldehydefixed tissue valve prostheses. Glutaraldehyde-fixed tissues such as bovine pericardium and porcine heart valves are the commonly used base materials for the construction of bioprosthetic heart valves as substitutes in humans. However, premature degradation limits valve durability, especially in younger patients. Cell-coated blood-contacting surfaces of glutaraldehyde-fixed biological heart valves are hypothesized to improve biocompatibility and durability. ${ }^{1}$ The toxicity of glutaraldehyde, however, prevents biological coating if not partially detoxified and preseeded by fibroblasts followed by endothelial cell seeding in vitro. ${ }^{1}$

Titanium has proven itself to be the leading structured metallic biomaterial for 50 years. ${ }^{2}$ Theoretically, surface coating of the less biocompatible glutaraldehyde-treated tissue with titanium may open new avenues for improving biocompatibility. However, a titanium coating on biomaterials seemed not to be possible until now owing to the high temperatures needed for commonly used sputtering techniques. Therefore, a coating method was applied for a biological substrate, using a plasma-activated chemical vapor deposition with the gaseous precursor TMT (tetrakisdimethyl-amidotitanium, $\left.\mathrm{Ti}\left[\mathrm{N}\left(\mathrm{CH}_{3}\right)_{2}\right]_{4}\right)$ at low pressure and temperatures of $30^{\circ} \mathrm{C}$ to $35^{\circ} \mathrm{C}$. $^{3}$ In addition to titanium coating, a technique for partial detoxification with citric acid (CA10\%), as described elsewhere, ${ }^{1}$ was applied on self-prepared glutaraldehyde-fixed $(0.25 \%$ over 24 hours) aortic porcine valves.

\section{HETEROTOPIC AORTIC VALVE IMPLANT}

Implantation of a preseeded aortic valve in the descending aortic position in a sheep model without extracorporal circulation has already been described. ${ }^{1}$ We used a male Bore goat with a weight of $83 \mathrm{~kg}$ (ethical accreditation of the

From the Klinik für Herzchirurgie, UK-SH Campus Lübeck, Lübeck, ${ }^{a}$ GfE Medizintechnik GmbH, Nürnberg, ${ }^{b}$ Klinik und Institut für Anästhesiologie, ${ }^{\mathrm{c}}$ Institut für Anatomie, Universität zu Lübeck, Lübeck, Germany. ${ }^{\text {d }}$

Received for publication Sept 30, 2008; revisions received Nov 5, 2008; accepted for publication Nov 24, 2008.

Address for reprints: Norbert W. Guldner, MD, Klinik für Herzchirurgie, Universitätsklinikum Schleswig-Holstein, Campus Lübeck, Ratzeburger Allee 160, D- 23538 Lübeck, Germany (E-mail: guldner@uni-luebeck.de).

J Thorac Cardiovasc Surg 2009; 138:248-50

$0022-5223 / \$ 36.00$

Copyright (c) 2009 by The American Association for Thoracic Surgery doi:10.1016/j.jtcvs.2008.11.045

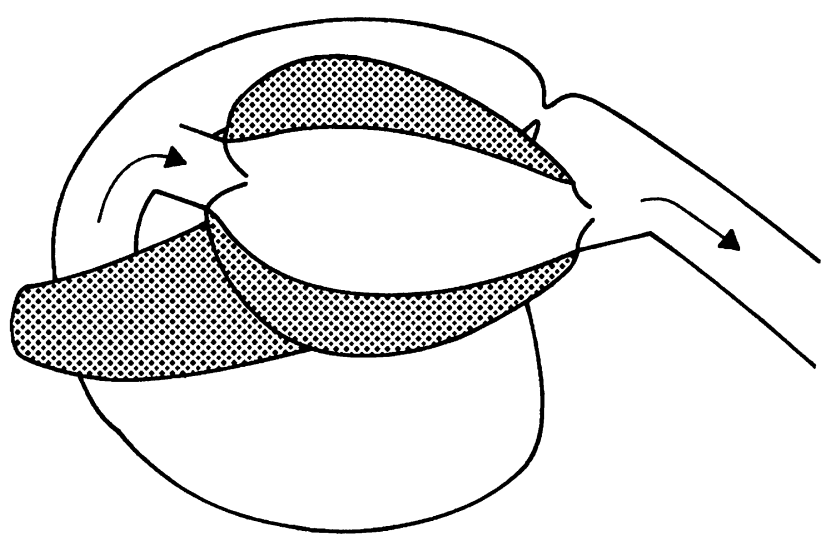

FIGURE 1. Valve topography within the inflow and outflow part of the pumping chamber of a biomechanical heart, a muscular blood pump within the aorto-aortic position.

Ethics Committees for Animals in Kiel, V 362-72241.1226) to create a muscular blood pump in the descending aortic position (Figure 1). We integrated two glutaraldehyde-fixed, titanium-coated and detoxified porcine heart valves into the inflow and outflow part of the pumping chamber of this muscular blood pump, the biomechanical heart. ${ }^{4}$ This skeletal muscle ventricle pumped with a frequency of 20 beats/ min. After 6 months of pumping, the valves were removed from the pumping chamber for histologic analysis.

\section{HISTOLOGIC AND IMMUNOHISTOCHEMICAL STAINING}

Valve leaflets from the two valves were embedded in paraffin, cut into sections of $4 \mu \mathrm{m}$, and treated with a hematoxylin-eosin stain (Figure 2, B). Other sections were treated using polyclonal antibodies against factor VIII (Dako, Hamburg, Germany). After incubation with primary antibody, an anti-rabbit immunoglobulin $\mathrm{G}$ antibody was applied that was conjugated with alkaline phosphatase (Sigma-Steinheim, Germany). Finally, substrate incubation (naphtole/neofuchsine, Dako) and counterstaining with hemalaun were performed (Figure 2, C). Others samples were conjugated with the fluorescence marker fluorescein isothiocyanate porc anti-rabbit immunoglobulin G (Dako,) and diluted 1:50 in $10 \%$ human serum (Figure 2, D). Cell nuclei were counterstained with propidium iodide (Sigma-Steinheim, Germany) diluted 1:500 in phosphate-buffered solution (Dako, 

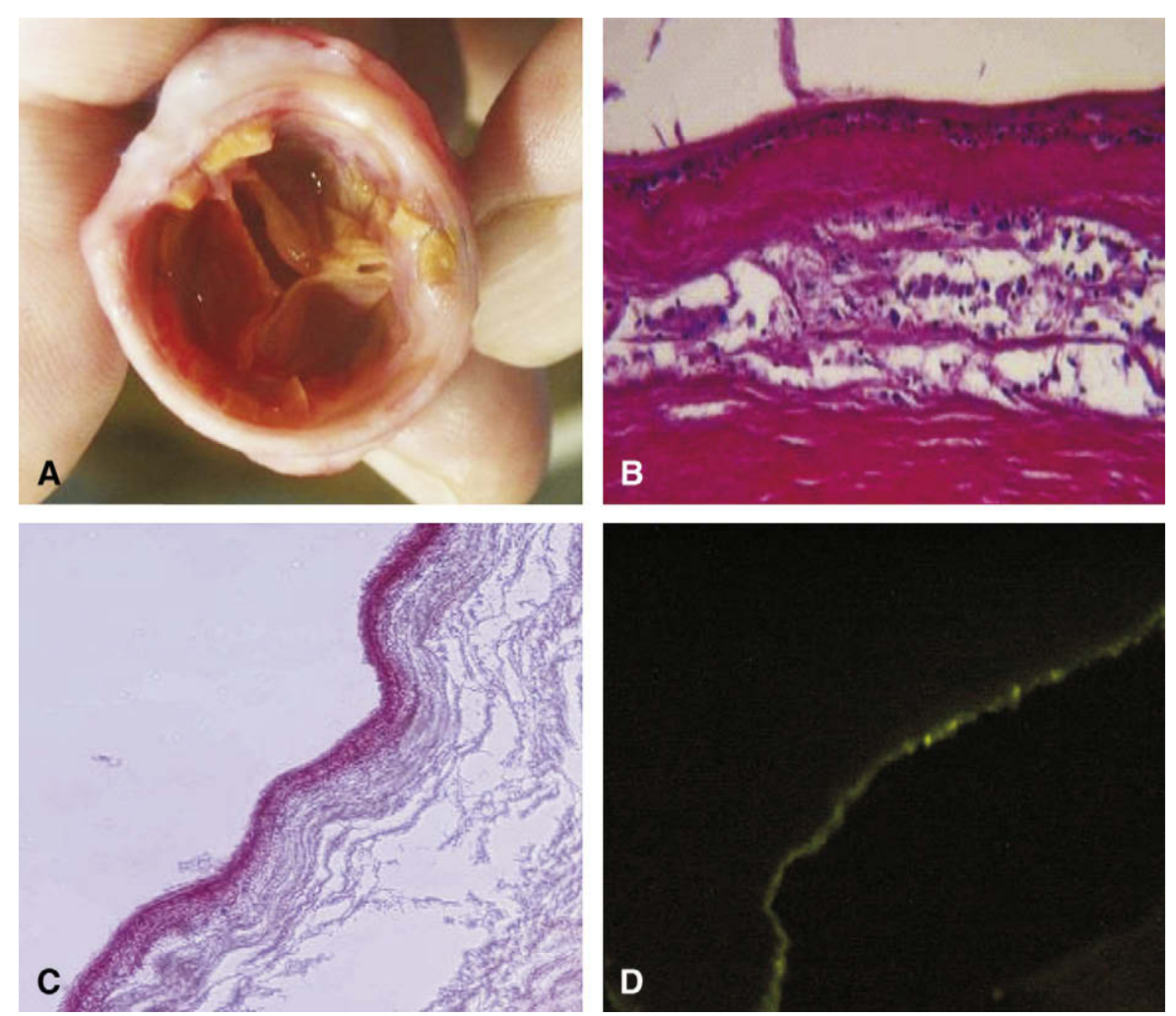

FIGURE 2. Porcine detoxified and titanized aortic valve, having been implanted heterotopically within the aorta of a goat for 6 months, is in good condition (A). Cross section of a valve leaflet (B) shows recellularization of the blood-contacting surface (hematoxylin-eosin stain, magnification $20 \times$ ). Immunhistochemic staining against von Willebrand factor proves endothelialization of the blood-contacting surface, labeled with alkaline phosphatase (C) and visualized with immunofluorescence (D).

Hamburg, Germany). Photographic documentation was performed by using a magnification of $20 \times$.

\section{RESULTS}

Porcine titanized and detoxified aortic valves, having been implanted heterotopically within the systemic circulation of a goat for 6 months, were macroscopically well (Figure 2, A) and showed a normal opening and closure behavior. Cross section of a valve leaflet (Figure 2, B) demonstrated a cell-seeding of its blood-contacting surface (hematoxylin-eosin staining, magnification $20 \times$ ). Immunhistochemical staining against factor VIII proved a complete endothelialization of the total blood-contacting surface, labeled with alkaline phosphatase (Figure 2, $C$ ) and visualized with immunofluorescence (Figure 2,D).

\section{CONCLUSIONS}

Tissue engineering of heart valves is considered to be a hopeful concept to generate improved substitute bioprostheses. However, because there is yet no realizable blueprint to construct semilunar valves,${ }^{5}$ our aim was to refine conventional, proven bioprostheses using innovative nanotechnologies such as plasma deposition with the most biocompatible titanium coating in combination with chemical agents like citric acid and aldehyde dehydrogenase. This procedure has been shown to support detoxification and endothelialization of biological glutaraldehyde-fixed tissue.

This newly developed method combining tissue engineering with nanotechnology, including a titanium coating, created the first self-seeded glutaraldehyde-fixed biological heart valve within the circulation under arterial pressure. It might open a new field of research for developing new heart valves with improved durability and function. This new technology might also be appropriate for creating endothelialized vascular prostheses with small diameters and the potential of a self-seeding blood-contacting surface accompanied by a hypothesized high patency rate, in aorta-coronary bypass surgery as well as in peripheral limb surgery. Further large animal studies are necessary to approve this procedure to increase durability and function in biological glutaraldehyde-fixed heart valves over several decades and avoid reoperations caused by valve degeneration.

\section{References}

1. Gulbins H, Pritisanac A, Piper K. Successful endothelialization of porcine glutaraldehyde-fixed aortic valves in a heterotopic sheep model. Ann Thorac Surg. 2006;81:1472-9. 
2. Köckerling F, Scheidbach P, Tannapfel A, Schmidt U, Lippert H. Influence of titanium coating on the biocompatibility of a heavyweight polypropylene mesh. Eur Surg Res. 2004;36:313-7.

3. Grill A. Cold plasma in materials fabrication: from fundamentals to applications. New York: IEEE Press; 1994. p. 1-416.
4. Guldner NW, Klapproth P, Großherr M, Rumpel E, Noel R, Sievers HH. Biomechanical hearts: muscular blood pumps, performed in a one-step operation, and trained under support of clenbuterol. Circulation. 2001;104:717-22.

5. Sievers HH. In vivo tissue engineering an autologous semilunar biovalve: can we get what we want? J Thorac Cardiovasc Surg. 2007;134:20-2. 